\title{
Influencia de tres tipos de vegetación ribereña en el control de la erosión fluvial en Pantanos de Centla, México
}

\author{
Alejandra Sepúlveda-Lozada ${ }^{1}$, Violette Geissen ${ }^{1}$, Susana Ochoa-Gaona ${ }^{1}$, Aarón Jarquín- \\ Sánchez ${ }^{1}$, Simón Hernández de la Cruz ${ }^{1}$, Edward Capetillo ${ }^{2}$ \& Luis Felipe Zamora-Cornelio ${ }^{1}$ \\ 1. El Colegio de la Frontera Sur, Administración de Correos 2, Apartado Postal 1042, 86100 Villahermosa, Tabasco, \\ México; vgeissen@web.de, aleja.sepulveda@gmail.com, sochoa@ecosur.mx, ajarquin@ecosur.mx, simon180277@ \\ hotmail.com,zamlf@yahoo.com.mx \\ 2. Universidad Tecnológica de Tabasco. Carretera Villahermosa-Teapa, Km. 14+600, Fraccionamiento Parrilla, Parrilla \\ II, Centro, Tabasco, C.P. 86280; golead_091087@hotmail.com
}

\section{Recibido 28-I-2009. Corregido 01-V-2009. Aceptado 02-VI-2009.}

\begin{abstract}
Influence of three types of riparian vegetation on fluvial erosion control in Pantanos de Centla, Mexico. Wetlands constitute very important ecological areas. The aim of this study was to quantify the soil losses due to fluvial erosion from 2006 to 2008 in two riverbanks under three types of vegetal coverage dominated by Haematoxylum campechianum, Dalbergia brownei and Brachiaria mutica, in the Pantanos de Centla Biosphere Reserve, SE Mexico. The relationship between the texture, organic matter and $\mathrm{pH}$ of soils and soil losses was evaluated. We used erosion sticks to estimate soil losses in 18 plots (three plots per type, three vegetation types, two riverbanks). Soil loss decreased in this order: H. campechianum $>$ B. mutica $>$ D. brownei indicating that $D$. brownei scrubland has the most potential to retain soil. The higher erosive impact within $H$. campechianum sites can be related with the low density of these trees in the study areas, as well as the lack of association with other types of vegetation that could reinforce the rooting of the soil profile. Furthermore, soil losses in $H$. campechianum sites were dependent on soil texture. The soils under this type of vegetal coverage were mainly sandy, which are more vulnerable to the erosive action in comparison with fine textured soils or soils with higher clay content, like the ones found in D. brownei and B. mutica sites. Soil losses of $100 \%$ in the second year ( $B$. mutica plots) can be attributed to the distribution of roots in the upper soil layer and also to livestock management along riverbanks. This study recognizes the importance of $D$. brownei scrublands in riverbank soil retention. Nevertheless it is necessary to consider the role of an entire vegetal community in future research. Rev. Biol. Trop. 57 (4): 1153-1163. Epub 2009 December 01.
\end{abstract}

Key words: fluvial erosion, riverine vegetation, riverbanks, soil losses.

Los humedales son sistemas naturales definidos por la Convención RAMSAR (Anónimo 1999) como extensiones de marismas, pantanos, turberas o superficies cubiertas de agua, ya sea estancada o corriente, dulce o salobre. Comprenden también las zonas de bordes fluviales o de costa adyacentes al humedal, así como las islas o las extensiones de agua marina que se encuentran dentro del mismo. En todos los humedales el suelo o sustrato está periódicamente saturado o cubierto de agua, siendo éste el factor dominante que determina la naturaleza del desarrollo del suelo y del tipo de comunidades de plantas (especies hidrófilas) y animales que los habitan (Carrera \& de la Fuente 2003). A pesar de su gran importancia, los humedales están seriamente amenazados. La deforestación en países tropicales en las últimas décadas ha causado severas pérdidas de suelos en áreas montañosas y un aumento en la tasa de sedimentos transportados por los ríos (Geissen et al. 2007). Los sedimentos 
provenientes de bancos ribereños pueden llegar a representar hasta el $85 \%$ de la carga sedimentaria en una cuenca (Simon \& Collison 2002). La erosión fluvial también contribuye de manera importante en el arrastre de sedimentos provenientes de los bordes de los ríos. Este tipo de erosión consiste en el desprendimiento de partículas o agregados del suelo de los bordes de ríos o arroyos por el flujo de agua que, junto con las fallas de los bancos (colapsos físicos generados por la inestabilidad de los suelos) produce el deterioro de las áreas de bordes fluviales (Lawler et al. 1997, Wynn 2006). La erosión de los bancos ribereños está determinada por el tamaño del canal, el caudal, la velocidad de la corriente y el régimen de transporte (Simon \& Rinaldi 2000, Benda et al. 2004, Couper 2004); así como por características de los suelos tales como el tamaño de partículas, densidad, contenido y tipo de arcilla, contenido de materia orgánica y porosidad (Grissinger 1982). Por otro lado, la vegetación ribereña además de los beneficios que genera en cuanto a los hábitats y al microclima y a su papel en la calidad del agua, tiene un impacto importante en la estabilidad de los bancos ribereños y en su morfología (Wynn 2006). También influye indirectamente sobre la erosión de los bancos de ríos al cambiar ciertas propiedades de los suelos, tales como la materia orgánica, la estabilidad de agregados y la densidad real (Mamo \& Bubenzer 2001a, 2001b); reduce la turbulencia generada cerca de los bordes y debilita corrientes secundarias, reduciendo así los impactos por erosión fluvial (Thorne \& Furbish 1995). El grado de dispersión de la vegetación a lo largo de los ríos es determinante en la distribución del estrés hidráulico (Pizzuto \& Mecklenburg 1989), de hecho se ha encontrado que los bordes de ríos con presencia de especies arbóreas, presentan la mitad de impactos por erosión que las zonas ribereñas con vegetación dispersa (Odgaard 1987). También se ha determinado una relación lineal entre la biomasa de raíces de vegetación ribereña y la resistencia del suelo al esfuerzo cortante (shear strength); según Micheli \& Kirchner (2002) la vegetación ribereña incrementa hasta $800 \%$ dicha resistencia. La densidad y la distribución de raíces de vegetación herbácea y leñosa tienen implicaciones en la erosión fluvial y en la estabilidad de los bancos ribereños. Generalmente, la vegetación herbácea presenta una alta densidad de raíces muy finas (diámetros $<0.5 \mathrm{~mm}$ ) en los primeros $30 \mathrm{~cm}$ de la superficie del suelo (Simon \& Collison 2002, Wynn et al. 2004), mientras que las raíces de especies leñosas con diámetros $>0.5 \mathrm{~mm}$ se distribuyen más ampliamente en el perfil de suelo (Wynn 2006). A pesar de la resistencia estructural que puede conferir la vegetación herbácea a los suelos debida al gran número de raíces finas, la distribución de las mismas en la parte superficial del suelo no protege áreas profundas del perfil que pueden estar sujetas a un fuerte estrés hidráulico (Murgatroyd \& Ternan 1983, Davies-Colley 1997, Trimble 1997, Lyons et al. 2000, Simon \& Collison 2002, Wynn \& Mostaghimi 2006).

Algunos estudios han demostrado que la resistencia a la erosión tiene una relación directa con la densidad de raíces $>0.5 \mathrm{~mm}$ de diámetro, por lo que la vegetación leñosa puede proveer mejor protección contra la erosión en bancos ribereños. Zaimes et al. (2005) describen que las pérdidas por erosión fluvial son significativamente más altas en cauces con pasto que con otro tipo de vegetación ribereña. Lyons et al. (2000) confirman estos resultados. Las zonas ribereñas perturbadas (zonas desnudas, con hundimientos y con raíces expuestas) son más vulnerables ante la erosión fluvial debido al socavamiento de material por debajo de la zona de reforzamiento de raíces que las áreas de vegetación leñosa o herbácea, o las zonas de vegetación mixta. Las pérdidas de suelo pueden ser muy altas, especialmente en sitios de clima tropical con fuertes lluvias y altas velocidades de corriente (USDA-NRCS 1998, Micheli \& Kirchner 2002).

Por lo anterior y debido a que se reportan pocos datos cuantitativos sobre los efectos de la vegetación en la estabilidad de bancos ribereños, es necesario el entendimiento de los procesos involucrados en los cambios de los bordes de ríos y de los efectos de la vegetación 
en esos procesos para llevar a cabo un adecuado manejo y conservación de los bordes de los ríos (Wynn 2006). El objetivo del presente trabajo fue cuantificar las pérdidas de suelo por erosión fluvial en dos tipos de cauces ribereños, bajo la influencia de tres tipos de cobertura vegetal adaptada a condiciones inundables en la Reserva de la Biosfera Pantanos de Centla: remanentes perturbados de selva baja de palo tinto o tintal (Haematoxylum campechianum L.), matorrales de muco o mucal (Dalbergia brownei Jacq. Schinz) y pastizales introducidos de pasto pará (Brachiaria mutica Forssk. Stapf). También se evaluó la relación entre algunas propiedades físico-químicas de los suelos y la pérdida o retención de los suelos ribereños.

\section{MATERIALES Y MÉTODOS}

Área de estudio: En un período de dos años (2006-2008) se trabajó a orillas de un tramo de aproximadamente $2000 \mathrm{~m}$ de longitud y $210 \mathrm{~m}$ de ancho del río Grijalva (G) y del canal La Calzada (C) en un tramo de $1290 \mathrm{~m}$ de longitud y $17.8 \mathrm{~m}$ de ancho promedio. El río Grijalva se caracteriza por una corriente fuerte y gran flujo de agua, mientras que el canal La Calzada por una corriente más lenta y menor flujo de agua, y conecta al río Tabasquillo con el Grijalva. Ambos se encuentran dentro de la Reserva de la Biosfera Pantanos de Centla, ubicada en la Región Hidrológica Grijalva-Usumacinta en la zona costera del sur del Golfo de México entre las coordenadas geográficas $17^{\circ} 57^{\prime} 53^{\prime \prime}$ $18^{\circ} 39^{\prime} 03^{\prime \prime} \mathrm{N}$, y $92^{\circ} 06^{\prime} 39^{\prime \prime}-92^{\circ} 47^{\prime} 58^{\prime \prime} \mathrm{W}$ (INE 2000). En el área se diferencian tres estaciones climáticas: a) sequía de marzo a mayo; b) lluvias tropicales de junio a octubre, y c) "nortes" de noviembre a febrero, época en que dominan los vientos provenientes del norte, frecuentemente acompañados por lluvias (Vega 2005). La temperatura media anual en la zona varía de 26 a $28^{\circ} \mathrm{C}$ y la precipitación total anual de 1200 a $2000 \mathrm{~mm}$ (Arriaga et al. 2000). La precipitación total anual en los años de estudio fue de $1107.3 \mathrm{~mm}$ en 2006 y de $1663.2 \mathrm{~mm}$ en 2007 , con lluvias altas en el mes de octubre, que causaron intensas inundaciones en la región (CONAGUA 2006, 2007).

Los suelos del área de estudio son planos y de tipos Gleysol y Fluvisol (Arriaga et al. 2000). Los tipos de cobertura en los segmentos de los cauces estudiados están representados por árboles dispersos y en baja densidad de $H$. campechianum (palo tinto, T), matorrales de D. brownei (muco, M) y pastos, predominantemente B. mutica (pasto pará, E).

Características generales de los tres tipos de cobertura vegetal considerados en este estudio:

a. Tintales, en los que predomina H. campechianum, árbol de la familia Fabaceae que alcanza una altura de alrededor de $7 \mathrm{~m}$, presenta un tronco ramificado, copas extendidas y produce una sombra que localmente impide el desarrollo de un estrato herbáceo (Palacio Aponte et al. 2002). Este tipo de vegetación se encuentra comúnmente sobre sedimentos aluviales y coaluviales (Ascencio \& Maldonado 2002). En las zonas ribereñas evaluadas, se encuentran dispuestos en una fila al borde del río con árboles dispersos.

b. Matorral de muco, dominado por D. brownei, arbusto trepador de la familia Fabaceae (Moreno-Casasola et al. 2008), con una altura que varía entre 3 y $5 \mathrm{~m}$ y una densidad que no permite el paso de la luz (Reys \& Acosta 2003). Chaturvedi \& Das (2003) encontraron que la profundidad de raíces de Dalbergia sissoo alcanza los $2.5 \mathrm{~m}$ y la distribución de sus raíces es simétrica. En la zona de estudio esta especie forma masas densas de 2 a $3 \mathrm{~m}$ de ancho a lo largo de la orilla de los cuerpos de agua.

c. Pastizales inducidos de B. mutica, pasto nativo de África (conocido como pasto pará) de la familia Poaceae y ahora distribuido en los trópicos y subtrópicos en áreas ganaderas. Su potencial como pastura es reconocido desde hace unos 20 años en América tropical. Se le considera un pasto valioso para el control de la erosión, ya que genera una buena cubierta sobre el 
suelo; su sistema de raíces consta de una base estolonífera con raíces que se desarrollan de los nodos basales. El pasto pará es una especie esencialmente de lugares tropicales húmedos, pero tolera períodos de sequía de 4 o 5 meses (Warren 2001). Los productores lo establecen a la orilla de los bordes de los ríos, extendiéndose en fajas de 5 a $30 \mathrm{~m}$ de ancho.

Propiedades de los suelos: En abril de 2008 se obtuvieron muestras de suelo (de 0-10 y de $10-30 \mathrm{~cm}$ de profundidad) en los sitios de estudio para la caracterización de las siguientes propiedades: textura (Bouyoucos, SEMARNAT 2000), materia orgánica (MO; Walkley \& Black, SEMARNAT 2000) y pH (KCl 1M; SEMARNAT 2000).

Cálculo de pérdidas de suelo: En abril del 2006 se establecieron parcelas de $1 \mathrm{~m}$ de ancho por $2.7 \mathrm{~m}$ de largo para la medición de pérdidas de suelo por erosión fluvial en zonas con H. campechianum (T), D. brownei (M) y B. mutica (E) a orillas del canal La Calzada (C) y el río Grijalva $(G)$. Se establecieron tres parcelas en cada tipo de cobertura vegetal en cada uno de los cauces: el canal (C) y el río $(G)$, respectivamente.

Para la medición de la erosión, en abril de 2006 se colocaron en cada parcela 10 varillas metálicas colocadas a $0.30 \mathrm{~m}$ unas de otras y a $1 \mathrm{~m}$ de distancia del borde del cauce $\mathrm{C}$ y $\mathrm{G}$ (Fig. 1). Esta distancia, fue considerada como distancia inicial, y sirvió de base para los cálculos de pérdida de suelo en el 2007 y 2008. Para ello, se midió anualmente con un flexómetro la distancia del borde del cauce a cada varilla (distancia final $D f$, Fig. 1).

Para los cálculos de áreas de suelo restantes y pérdidas, generamos las siguientes fórmulas:

(1) $\mathrm{ASRP}=\sum\left[\left(D f_{1}+D f_{2}\right) / 2\right] \cdot 0.30+\left[\left(D f_{2}+D f_{3}\right) / 2\right] \cdot 0.30+$ $\ldots+\left[\left(D f_{9}+D f_{10}\right) / 2\right] \cdot 0.30$

(2) $\mathrm{ASPP}=2.7-\sum\left[\left(D f_{1}+D f_{2}\right) / 2\right] \cdot 0.30+$ $\left[\left(D f_{2}+D f_{3}\right) / 2\right] \cdot 0.30+\ldots+\left[\left(D f_{9}+D f_{10}\right) / 2\right] \cdot 0.30$ donde: $\mathrm{ASRP}=$ área de suelo restante por parcela en $\mathrm{m}^{2}, \mathrm{ASPP}=$ área de suelo perdida por parcela $\left(\mathrm{m}^{2}\right)$; y $D f_{1}-D f_{10}$ las distancias finales de cada varilla hacia el cauce (m). En ambas fórmulas, 0.30 representa la distancia entre varillas y 2.7 el área total $\left(\mathrm{en}^{2}\right)$ de las parcelas al inicio del estudio.

Los cálculos anteriores se transformaron a porcentajes mediante las siguientes fórmulas:

\section{(3) $\operatorname{ASRP}(\%)=(\mathrm{ASRP} \cdot 100) / 2.7$ \\ (4) $\operatorname{ASPP}(\%)=(\operatorname{ASPP} \cdot 100) / 2.7$}

Para la detección de normalidad en los datos generados se corrió la prueba de Kolmogorov-Smirnov. Con datos paramétricos se realizó un ANDEVA de un factor y se corrieron las pruebas post hoc T3 de Dunnett (homocedasticidad) y de Bonferroni (no homocedasticidad) (Zar 1984) para comparar la variabilidad entre los sitios de estudio. Se realizaron pruebas de Kruskal-Wallis seguidas de la prueba de MannWhitney para datos no paramétricos (Underwood 1981) y correlaciones de Pearson entre las propiedades del suelo y las pérdidas de suelo por erosión fluvial. Se hizo un gráfico de cajas anidado para contrastar las pérdidas de suelo bajo distintos tipos de cobertura vegetal en los dos tipos de cauce (mediana, $Q_{1}, Q_{3}$, valores mínimos y máximos) y un análisis de componentes principales (ACP's) con 3 variables (MO, contenido de arena y pérdida de suelo) y 18 puntos de muestreo en el período de estudio 2006-2008 para conocer la influencia de las variables en la variabilidad de los sitios.

\section{RESULTADOS}

Propiedades de los suelos: Los suelos dominantes en los sitios de estudio fueron migajoso-arcillosos y arcilloso-limosos (Cuadro, 1 con promedio de las profundidades $0-10$ y $10-30 \mathrm{~cm}$, las cuales no presentaron diferencias significativas en textura y $\mathrm{pH}$ ). Sin embargo, también se encontraron suelos francos, franco-arcillosos, franco-arcillo-limosos y franco-arcillo-arenosos en zonas con $\mathrm{T}$ en $\mathrm{C}$ y $\mathrm{G}$, así como suelos arcillo-limosos en sitios 


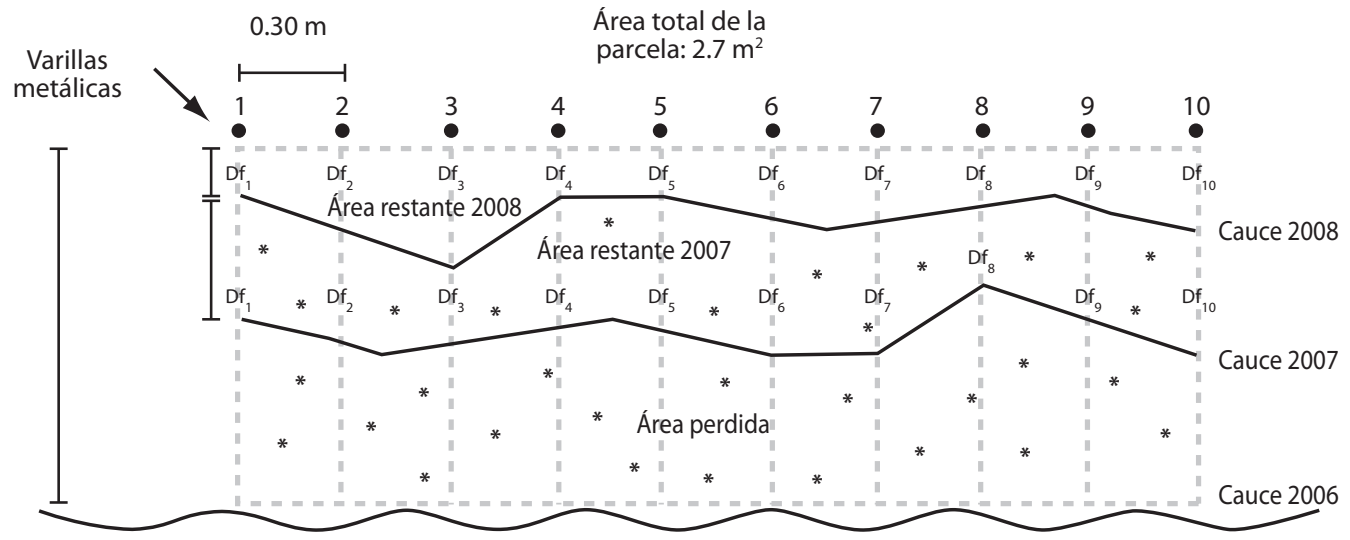

Fig. 1. Vista superior de las parcelas de medición para evaluar pérdidas de suelo por erosión fluvial.

Fig. 1. Top view of the plots measured to assess soil losses by water erosion.

\section{CUADRO 1}

Propiedades físico-químicas de suelos aledaños al río Grijalva $(G)$ y Canal La Calzada $(C)$, en sitios con palo tinto (T), muco (M) y pasto pará (E)

TABLE 1

Physical-chemical properties of soil adjacent to the Grijalva River (G) and the Channel La Calzada (C), at sites with Logwood (T), Brown's Indian rosewood (M) and para grass (E)

$\begin{array}{ccccccl}\text { Sitio } & \mathrm{pH}(\mathrm{KCl}) & \text { Materia orgánica (\%) } & \text { Arcilla (\%) } & \text { Limo (\%) } & \text { Arena (\%) } & \text { Clase textural } \\ \text { C-T } & 7.16 \pm 0.02 & 3.23 \pm 0.08 \mathrm{a} & 27.17 \pm 2.89 \mathrm{a} & 36.06 \pm 3.40 & 36.78 \pm 13.1 \mathrm{~b} & \text { Migajosa-arcillosa } \\ \mathrm{C}-\mathrm{M} & 6.67 \pm 0.65 & 7.79 \pm 1.55 \mathrm{c} & 43.97 \pm 5.51 \mathrm{~b} & 44.61 \pm 4.87 & 11.42 \pm 0.65 \mathrm{a} & \text { Arcillosa-limosa } \\ \mathrm{C}-\mathrm{E} & 6.76 \pm 0.35 & 8.22 \pm 0.91 \mathrm{c} & 45.33 \pm 5.61 \mathrm{~b} & 44.06 \pm 1.02 & 10.61 \pm 5.06 \mathrm{a} & \text { Arcillosa-limosa } \\ \mathrm{R}-\mathrm{T} & 7.22 \pm 0.01 & 2.85 \pm 0.45 \mathrm{a} & 30.33 \pm 7.22 \mathrm{a} & 37.06 \pm 8.11 & 32.61 \pm 15.3 \mathrm{~b} & \text { Migajosa-arcillosa } \\ \mathrm{R}-\mathrm{M} & 7.13 \pm 0.02 & 3.70 \pm 0.60 \mathrm{a} & 40.22 \pm 0.86 \mathrm{~b} & 48.39 \pm 1.84 & 11.39 \pm 2.28 \mathrm{a} & \text { Arcillosa-limosa } \\ \text { R-E } & 7.12 \pm 0.09 & 5.03 \pm 0.34 \mathrm{~b} & 40.00 \pm 1.20 \mathrm{~b} & 47.78 \pm 0.69 & 12.22 \pm 0.69 \mathrm{a} & \text { Arcillosa-limosa }\end{array}$

Diferencias significativas $(\mathrm{p}<0.05)$ entre los sitios: $\mathrm{a}<\mathrm{b}<\mathrm{c}$.

con $\mathrm{M}$ y $\mathrm{E}$ en $\mathrm{C}$ y $\mathrm{G}$. $\mathrm{El} \mathrm{pH}$ de todos los suelos fue neutro (6.67 a 7.22). Los suelos con $\mathrm{pH}$ más bajo, presentaron los contenidos más altos de MO (Cuadro 1), relación que se reafirma con la correlación negativa entre el $\mathrm{pH}$ y la $\mathrm{MO}(\mathrm{r}$ de Pearson=-0.72, $\mathrm{p}=0.01$ ). La MO varió de 2.85 a $8.22 \%$ (Cuadro 1), y presentó contenidos significativamente más altos en la capa de 0-10 cm en comparación con la capa de 10-30 cm de profundidad. Los contenidos más bajos se presentaron en zonas con vegetación de $\mathrm{T}$ y los más altos en zonas con E y M (Cuadro 1). La $\mathrm{MO}$ y la arcilla presentaron correlación positiva
( $\mathrm{r}$ de Pearson $=0.73, \mathrm{p}=0.01$ ). Los sitios con bajos contenidos de MO (2.85-3.23\%) presentaron los más altos contenidos de arena (32.6-36.8\% en áreas de $\mathrm{T}$ en $\mathrm{C}$ y $\mathrm{G}$ ), lo que se reflejó en una correlación negativa entre la arcilla y arena ( $\mathrm{r}$ de Pearson $=-0.90, p=0.01$ ) y entre la materia orgánica y arena ( $\mathrm{r}$ de Pearson $=-0.55, \mathrm{p}=0.05)$. Los sitios con $\mathrm{M}$ y E presentaron los contenidos más altos de arcilla y MO (Cuadro 1).

Pérdida de suelo y factores influyentes: En el primer año de estudio (2006-2007), tanto en $\mathrm{C}$ como en $\mathrm{G}$ se observó que las zonas con $\mathrm{T}$ 
presentaron pérdidas significativamente mayores de suelo (de $57.5 \%$ en $\mathrm{C}$ a $73.5 \%$ en $\mathrm{G}$ ) con relación a las zonas con $\mathrm{E}$ y $\mathrm{M}(17-66 \%$ en $\mathrm{C}$ y $\mathrm{G}$, y $6-32 \%$ en $\mathrm{G}$ y $\mathrm{C}$, respectivamente; Fig. 2A). Las pérdidas significativamente más bajas en $\mathrm{C}$ se presentaron en zonas con E (17\%), mientras que en $\mathrm{G}$ se presentaron en zonas con $\mathrm{M}$ (6\%, Fig. 2A).

$\mathrm{Al}$ considerar las pérdidas acumuladas del primer y segundo período de estudio (20062008), las zonas con $\mathrm{T}$ y E presentaron las mayores pérdidas (77-100\%), mientras que las zonas con $\mathrm{M}$ en ambos sitios siguieron presentando las menores pérdidas de suelo $(7.5 \%$ en G y $64 \%$ en C, Fig. 2B). La tendencia en cuanto a pérdidas de suelo por tipo de cobertura vegetal se presentó en el siguiente orden: $\mathrm{T}>\mathrm{E}>\mathrm{M}$ (Fig. 2)

Para el período 2006-2008 se observó que el contenido de materia orgánica y de arena explicaron un $56.5 \%$ de la variabilidad de los sitios (primer componente principal de la Fig. 3). Las pérdidas en G-T se relacionaron positivamente con el contenido de arena y negativamente con el contenido de MO. En C-M, hubo una relación negativa entre el contenido de $\mathrm{MO}$ y las pérdidas de suelo. En C-T, C-E, G-E y G-M no se encontró relación entre las pérdidas de suelos con la MO o la arena (Fig. 3). El segundo componente representa las pérdidas de suelos en función de otros factores (quizá el efecto de la misma vegetación, entre otros no determinados en este estudio), explicó el 33.9\% de la variabilidad (Fig. 3).

\section{DISCUSIÓN}

Entre los tipos de vegetación estudiados, el matorral de D. brownei fue el de mayor potencial para la retención de suelos aledaños al río Grijalva y al Canal La Calzada en la Reserva de la Biosfera Pantanos de Centla. Las raíces de Dalbergia se presentan cerca del tronco y se ramifican desde su base en forma de abanico y
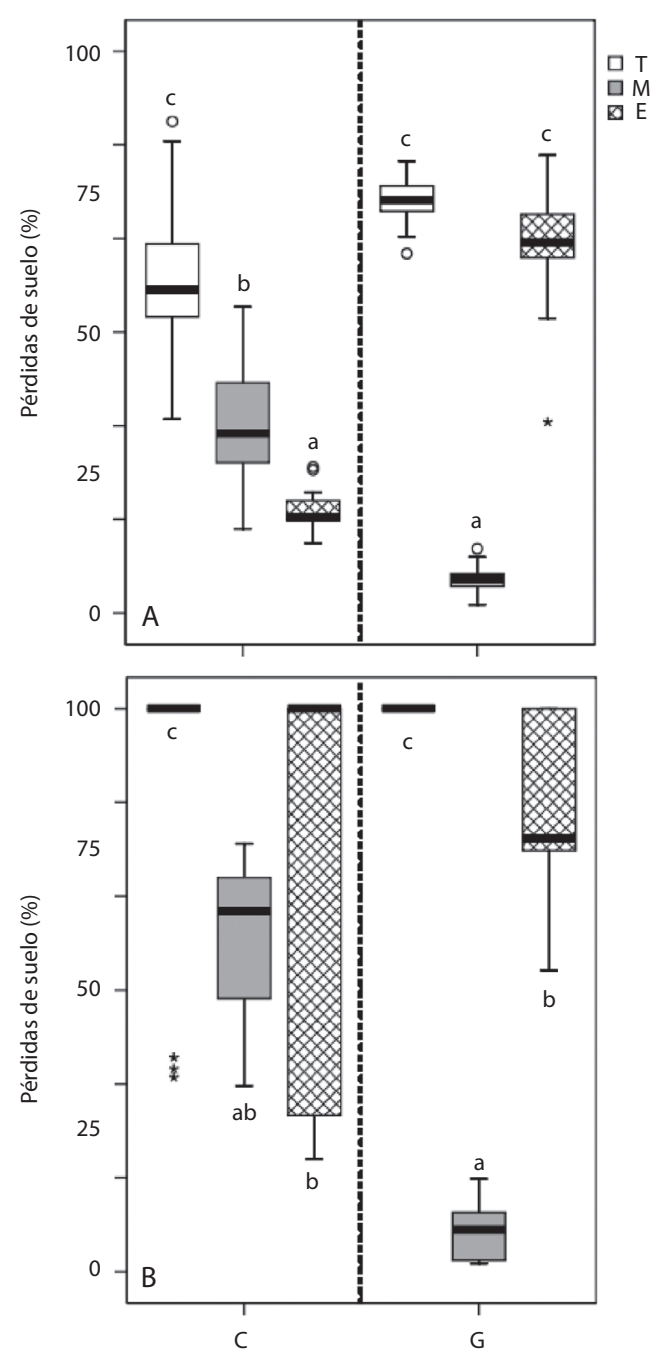

Fig. 2. Pérdidas de suelo por erosión fluvial en el Canal La Calzada (C) y río Grijalva (G) en zonas con vegetación de T, M y E. (A) 2006-2007 y (B) 2006-2008; $a<a b<b<c$ : diferencias significativas $(\mathrm{p}<0.05)$ dentro y entre los sitios. Mediana, $\mathrm{Q}_{1}, \mathrm{Q}_{3}$, valores mínimos y máximos.

Fig 2. Loss of soil by water erosion in the Channel La Calzada (C) and Grijalva River $(G)$ in areas with vegetation of T, M and E. (A) 2006-2007 and (B) 2006-2008; $\mathrm{a}<\mathrm{ab}<\mathrm{b}<\mathrm{c}$ : significant differences $(\mathrm{p}<0.05)$ within and between sites. Median, Q1, Q3, minimum and maximum values. 


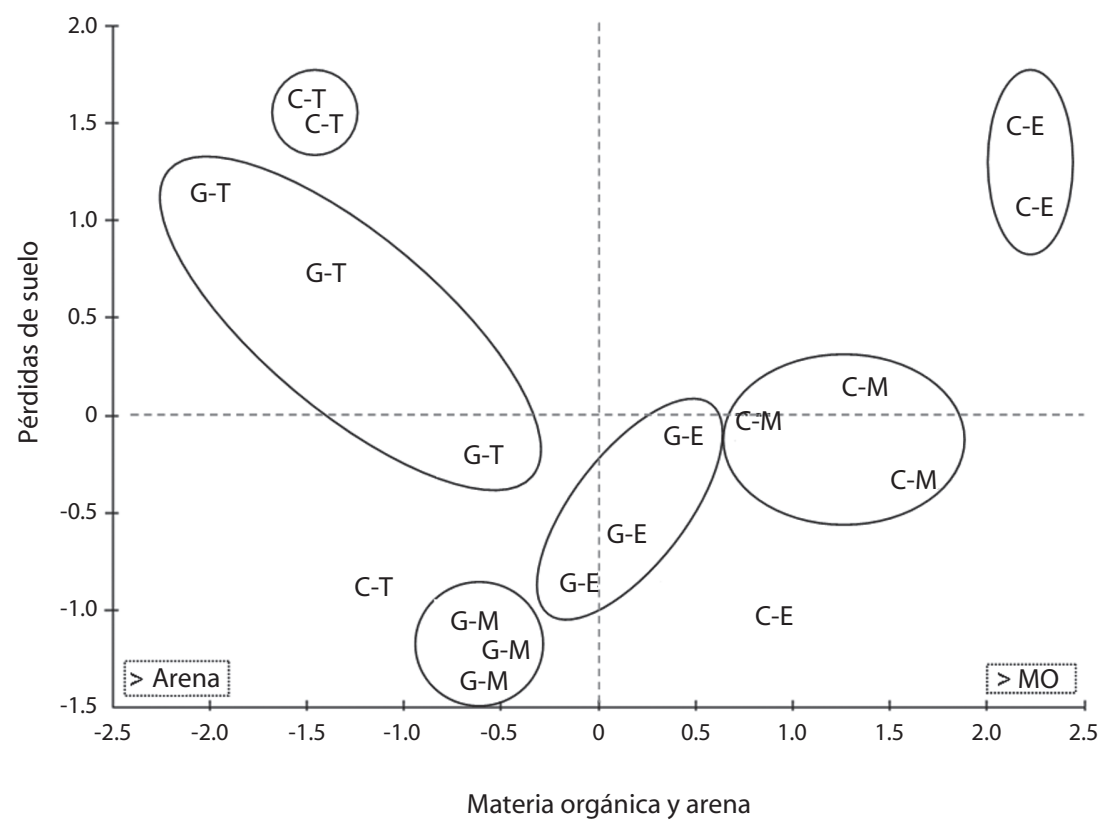

Fig. 3. Componentes principales extraídos de las propiedades físico-químicas de los suelos y pérdida de suelos (2006-2008). CP1 representado por el contenido de materia orgánica y arena, CP2 representado por la pérdida de suelo. C: Canal La Calzada, G: río Grijalva, T: palo tinto, M: muco, E: pasto pará.

Fig 3. Principal components extracted from the physical and chemical properties of soil and soil loss (2006-2008). CP1 represented by organic matter and sand content, CP2 represented by the loss of soil. C: Channel La Calzada, G: Grijalva River, T: Logwood, M: Brown's Indian rosewood, E: Para grass.

su dispersión horizontal comprende entre $1.1 \mathrm{y}$ 1.6 veces la proyección de la copa (Chaturvedi \& Das 2003), lo que puede relacionarse con una mayor retención de los suelos. De hecho, autores como Puri \& Swamy (1999) y von Carlowitz (1986) han recalcado el potencial de Dalbergia en la estabilización de paisajes erosionados y de bancos de ríos.

Pese a que la bioingeniería considera a $H$. campechianum como una especie adecuada para el control de la erosión de suelos (Clark et al. 1995), en este estudio las mayores pérdidas de suelo por erosión fluvial se presentaron en zonas con este tipo de vegetación. Observaciones en campo permitieron reconocer algunos rasgos de la morfología y disposición de las raíces de $H$. campechianum como el predominio de un sistema primario de raíces (característico de las Fabaceae, Burkart 1987) y una amplia distribución horizontal por encima de la superficie de agua, como ha sido reportado para Prosopis pallida (Tume et al. 1989, Singh \& Singh 1993). Sin embargo, por debajo de su sistema de raíces fue notorio el socavamiento de suelo por la corriente fluvial. Este mayor impacto erosivo en sitios con $H$. campechianum puede relacionarse con la disposición muy dispersa de los árboles en los sitios de estudio, así como por la ausencia de asociación con otros tipos de vegetación que reforzaran la estructura subterránea de sus raíces. Además de la influencia del tipo de vegetación, las pérdidas de suelo en zonas con $H$. campechianum fueron dependientes de la textura. Los suelos bajo este tipo de cubierta vegetal fueron principalmente arenosos, que son más susceptibles a procesos erosivos en comparación con suelos de texturas finas o con mayor contenido de arcilla (Park \& Egbert 2005). 
Aunque las pérdidas de suelo en sitios con $D$. brownei y $B$. mutica no fueron dependientes de la textura del suelo, el suelo de estos sitios presentó altos contenidos de arcilla, limo y materia orgánica. Estas características, confieren a los suelos mayor resistencia a la erosión debido a que las partículas finas de arcilla y limo se combinan con la materia orgánica para formar agregados con un buen grado de cohesión y estabilidad (Morgan 1986, Park \& Egbert 2005). El componente orgánico es de gran importancia ya que se ha observado que la erodabilidad del suelo disminuye linealmente cuando se incrementa el contenido de materia orgánica (Voroney et al. 1981). En este aspecto, la vegetación, y especialmente las especies fijadoras de nitrógeno, son claves en la mejora de algunas propiedades del suelo al aportar materia orgánica, así como al mantener e incrementar la fertilidad y estructura del suelo (Santana 2007). Dalbergia es fijadora de nitrógeno (Hines \& Eckman 1993) y por ello cobra un papel relevante en la contención de la erosión.

Las pérdidas del $100 \%$ de suelo en el segundo año por erosión fluvial en zonas con pasto pará (B. mutica) concuerdan con los estudios de Lyons et al. (2000), Micheli \& Kirchner (2002) y Zaimes et al. (2005). Esto puede deberse a que las áreas profundas del perfil no se ven protegidas, a pesar de la resistencia estructural que los pastos brindan a los suelos con su sistema de raíces, debido a su distribución superficial (Murgatroyd \& Ternan 1983, Davies-Colley 1997, Trimble 1997, Lyons et al. 2000, Simon \& Collison 2002, Wynn \& Mostaghimi 2006).

Otro de los factores que pueden contribuir a la degradación y pérdida de suelos ribereños en el área de estudio es la actividad ganadera. Las zonas con pasto pará son áreas sujetas al pastoreo y por lo tanto al pisoteo, lo cual altera la estabilidad del suelo. El pastoreo en áreas ribereñas ha alterado el ciclo hidrológico natural y ha acelerado la erosión de los bancos ribereños. En zonas con este tipo de actividad, la erosión de los bancos puede contribuir entre un 50 y un $90 \%$ a la carga de sedimentos y fósforo al ambiente acuático (Zaimes et al. 2005).
Además de la influencia de la morfología de la vegetación, de las mismas características de los suelos y de los posibles impactos de las actividades productivas, como la ganadería, en la retención de suelos en las márgenes de los ríos, el comportamiento climático en la zona en una escala más amplia regula los procesos de erosión y sedimentación. Dado que no existen otros datos de erosión fluvial para la zona, es difícil definir la influencia concreta de las lluvias en la dinámica de erosión fluvial, por lo que resulta importante estudiar las interrelaciones entre los procesos de erosión, sedimentación, características geomorfológicas, vegetación y la variación en el aporte del caudal de los ríos con métodos más rigurosos para analizar estas relaciones.

Con este estudio se puede reconocer la importancia de los matorrales de D. brownei en la retención de suelos ribereños en Pantanos de Centla. H. campechianum y B. mutica no fueron tan eficientes como D. brownei en el control de erosión fluvial, sin embargo, hay que considerar que la influencia de solo un tipo de cobertura en la retención de suelos aledaños a ríos puede no ser tan significativa como el papel de una comunidad vegetal, en la que su composición y densidad, así como su diversificación en morfología (principalmente de raíces) tenga un papel importante. Aunado a esto, resulta importante llevar a cabo estudios en la zona acerca de la morfología, densidad y distribución de raíces de vegetación herbácea y leñosa, ya que esto tiene implicaciones en la erosión fluvial y en la estabilidad de los bancos ribereños.

Una alternativa de manejo para el aminoramiento y control de la erosión en zonas de pastoreo a la orilla de los ríos es la incorporación de especies leñosas perennes, lo cual permitiría incrementar la fertilidad del suelo, mejorar su estructura y disminuir los procesos de erosión. Estos resultados han sido explicados por el mayor reciclaje de nutrientes que ocurre, la fijación de $\mathrm{N}$, la profundización de las raíces de los árboles, la mayor actividad de la macro y micro fauna y el control de la erosión (Mahecha 2002). 
Finalmente, una mejor comprensión de la relación entre la vegetación y la estabilidad de los bancos ribereños conducirá a la toma de mejores decisiones en lo que se refiere a estrategias de conservación y restauración.

\section{AGRADECIMIENTOS}

A Pablo Martínez Zurimendi por la revisión de la redacción del manuscrito y a Alejandro Ponce Mendoza por sus comentarios. Aarón de Jesús Rueda Torruco colaboró en la toma de datos. A los productores de Tabasquillo municipio de Centla por permitirnos el acceso a sus parcelas para el establecimiento del experimento. A la CONANP por el permiso para la realización del trabajo en la Reserva Pantanos de Centla. El FONSEC CONACYTCONAFOR financió el proyecto Evaluación de la germinación y establecimiento inicial de árboles nativos con potencial para la restauración de humedales con número de registro 14794, Fondo S0002-2005-2. ECOSUR apoyó con infraestructura.

\section{RESUMEN}

En el presente trabajo se cuantificaron las pérdidas de suelo por erosión fluvial (2006-2008) en dos cauces ribereños con cobertura de Haematoxylum campechianum, Dalbergia brownei y Brachiaria mutica en la Reserva de la Biosfera Pantanos de Centla, SE de México. Se evaluó la relación entre la textura, materia orgánica y $\mathrm{pH}$ de los suelos con la pérdida de los mismos. Las pérdidas de suelo se estimaron en 18 parcelas con varillas de erosión. La principal tendencia de las pérdidas de suelo por tipo de cobertura vegetal se dio en el orden $H$. campechianum $>B$. mutica $>$ D. brownei. El mayor impacto erosivo en sitios con H. campechianum se relacionó con la textura arenosa de los suelos y el grado de perturbación de los sitios (baja densidad de árboles, alta dispersión y falta de asociación con otros tipos de vegetación). Las pérdidas totales de suelo en parcelas con $B$. mutica al segundo año de estudio pueden relacionarse con la distribución superficial de las raíces de este pasto y con la actividad ganadera. Con este estudio se reconoce la importancia de los matorrales de $D$. brownei en la retención de suelos ribereños, sin embargo hay que considerar que la sola influencia de un tipo de cobertura vegetal puede no ser tan significativa como el papel de una comunidad vegetal, en la que su composición y densidad, así como su diversificación en morfología tenga un papel importante en la conservación de los suelos.
Palabras clave: bordes de río, erosión fluvial, pérdida de suelos, vegetación riparia.

\section{REFERENCIAS}

Arriaga, L., V. Aguilar \& J. Alcocer. 2000. Aguas continentales y diversidad biológica de México. Comisión Nacional para el Conocimiento y Uso de la Biodiversidad. México DF, México.

Ascencio, J.M. \& F. Maldonado. 2002. El jardín botánico universitario "José Narciso Rovirosa": un esfuerzo para la conservación de la flora tabasqueña. Kuxulkab 7: 34-47.

Benda, L., N.L. Poff, D. Miller, T. Dunne, G. Reeves, G. Pess \& M. Pollock. 2004. The network dynamics hypothesis: how channel networks structure riverine habitats. BioScience 54: 413-427.

Burkart, A. 1987. Leguminosas, p. 467-538. En M. Dimitri (ed). Enciclopedia Argentina de Agricultura y Jardinería. Tomo I. Descripción de plantas cultivadas. ACME, Buenos Aires, Argentina.

Carrera, E. \& G. de la Fuente. 2003. Inventario y clasificación de humedales en México. Parte 1. Ducks Unlimited de México, México DF, México.

Chaturvedi, O.P. \& D.K. Das. 2003. Studies on rooting patterns of 5-year-old important agroforestry tree species grown in North Bihar, India. Forest, Trees \& People 12: 329-339.

Clark, J., T. Foy \& J. Hellin. 1995. Vegetation and slopes. p. 184-189. En D.H. Barker (ed). Stabilisation, protection and ecology. Thomas Telford, Londres, Inglaterra.

CONAGUA. 2006. Observaciones climatológicas de la Estación Tres Brazos, Centla, Tabasco. Comisión Nacional del Agua. Gerencia Estatal Tabasco, México.

CONAGUA. 2007. Observaciones climatológicas de la Estación Tres Brazos, Centla, Tabasco. Comisión Nacional del Agua. Gerencia Estatal Tabasco, México.

Couper, P.R. 2004. Space and time in river bank erosion research: A review. Area 36: 387-103.

Davies-Colley, R.J. 1997. Stream channels are narrower in pasture than in forest. New. Zeal. J. Mar. Freshwater Res. 31: 599-608.

Geissen, V., C. Kampichler, J.J. López-de Llergo-Juárez \& A. Galindo-Alcántara. 2007. Superficial and 
subterranean soil erosion in Tabasco, tropical Mexico: development of a decision tree modeling approach. Geoderma 139: 277-287.

Grissinger, E.H. 1982. Bank erosion of cohesive materials, p. 273-287. En R.D. Hey, J.C. Bathurst, C.R. Thorne (eds). Gravel-bed Rivers. Wiley, Nueva York, Nueva York, EEUU.

Hines, D.A. \& K. Eckman. 1993. Indigenous multipurpose trees of Tanzania: uses and economic benefits for people. FAO. Documento Forestal. Roma, Italia.

INE. 2000. Programa de Manejo de la Reserva de la Biosfera Pantanos de Centla. Instituto Nacional de Ecología, México DF, México.

Lawler, D.M., C.R. Thorne \& J.M. Hooke. 1997. Bank erosion and instability, p. 138-172. En C.R. Thorne, R.D. Hey \& M.D. Newson (eds). Applied fluvial geomorphology for river engineering and management. Wiley, Nueva York, Nueva York, EEUU.

Lyons, J., S.W. Trimble \& L.K. Paine. 2000. Grass versus trees: Managing riparian areas to benefit streams of central North America. Water Resour. Bull. 36: 919-930.

Mahecha, L. 2002. El silvopastoreo: una alternativa de producción que disminuye el impacto ambiental de la ganadería bovina. Revista Colombiana de Ciencias Pecuarias 15: 226-231.

Mamo, M. \& G.D. Bubenzer. 2001a. Detachment rate, soil erodibility, and soil strength as influenced by living plant roots, Part I: Laboratory Study. Transactions of the ASAE 44: 1167-1174.

Mamo, M. \& G.D. Bubenzer. 2001b. Detachment rate, soil erodibility, and soil strength as influenced by living plant roots, Part II: field study. Transactions of the ASAE 44: 1175-1181.

Micheli, E.R. \& J.W. Kirchner. 2002. Effects of wet meadow riparian vegetation on streambank erosion. 2 . Measurements of vegetated bank strength and consequences for failure mechanics. Earth Surf. Process. 27: $687-697$.

Moreno-Casasola, P., H. López Rosas, D. Infante Mata, L.A. Peralta, A.C. Travieso-Bello \& B.G. Warner. 2008. Environmental and anthropogenic factors associated with coastal wetland differentiation in $\mathrm{La}$ Mancha, Veracruz, Mexico. Plant. Ecol. doi: 10.1007/ s11258-008-9400-7.

Morgan, R.P.C. 1986. Soil erosion and conservation. Longman Scientific \& Technical, Londres, Inglaterra.
Murgatroyd, A.L. \& J.L. Ternan. 1983. The impact of afforestation on stream bank erosion and channel form. Earth Surf. Process. 8: 357-370.

Odgaard, A.J. 1987. Streambank erosion along 2 rivers in Iowa. Water Resour. Res. 23: 1225-1236.

Palacio Aponte, A.G., R. Noriega Trejo \& P. Zamora Crescendo. 2002. Caracterización físico-geográfica del paisaje conocido como bajos inundables. El caso del área natural protegida Balamkín, Campeche. Investigaciones Geográficas 49: 57-73.

Park, S. \& S.L. Egbert. 2005. Assessment of soil erodibility indices for conservation reserve program lands in Southwestern Kansas using satellite imagery and GIS Techniques. Environ. Manag. 36: 886-898.

Pizzuto, J.E. \& T.S. Meckelnburg. 1989. Evaluation of a linear bank erosion equation. Water Resour. Res. 25: 1005-1013.

Puri, S. \& S.L. Swamy. 1999. Geographical variation in rooting ability of stem cuttings of Azadirachta indica and Dalbergia sissoo. Genet. Resour. Crop. Ev. 46: 29-36.

Reys, O.J. \& F. Acosta. 2003. Fitocenosis presentes en las áreas costeras del sur de la Sierra Maestra, Cuba. I. Comunidades con influencia marina. Foresta Veracruzana 5: 1-8.

Santana, M. 2007. Fijación biológica de nitrógeno por leguminosas arbóreas para sombra de café en Puerto Rico. Tesis de Maestría. Universidad de Puerto Rico, Mayaguez, Puerto Rico.

SEMARNAT. 2000. Norma Oficial Mexicana NOM-021RECNAT-2000. Segunda Sección. Secretaría de Medio Ambiente y Recursos Naturales, México.

Simon, A. \& A. Collison. 2002. Quantifying the mechanical and hydrologic effects of riparian vegetation on stream bank stability. Earth Surf. Process. 27: 527-546.

Simon, A. \& M. Rinaldi. 2000. Channel evolution in the loess area of the Midwestern United States. J. Am. Water. Resour. As. 36: 133-150.

Singh, G. \& N.T. Singh. 1993. Mesquite for the revegetation of salt lands. Bulletin No.18. Central Soil Salinity Research Institute, Karnal, Haryana, India.

Thorne, S.D. \& D.J. Furbish. 1995. Influences of coarse bank roughness on flow within a sharply curved river bend. Geomorphology 12: 241-257. 
Trimble, S.W. 1997. Stream channel erosion and change resulting from riparian forests. Geology 25: 467469.

Tume, Z.P., T.M. Cardenas \& C.W. Calderón. 1989. Efecto de la napa freática en el crecimiento y enraizamiento de Prosopis pallida en Piura. Rev. Forestal del Perú 16: $65-71$.

Underwood, A.J. 1981. Techniques of analysis of variance in experimental marine biology and ecology. Oceanogr. Mar. Biol. 19: 513-605.

USDA-NRCS. 1998. Erosion and sediment delivery. Field Office Technical Guide Notice No. IA-198. Department of Agriculture, Natural Resource Conservation Service, Des Moines, Iowa, EEUU.

Vega, M.A. 2005. Plan de Conservación para la Reserva de la Biosfera Pantanos de Centla y el Área de protección de flora y fauna Laguna de Términos. PRONATURA Península de Yucatán/Programa Costero, México.

von Carlowitz, P.G. 1986. Recommendations for the design and establishment of demonstration trials at the Ethiopian Centre for community forestry and soil conservation. A model for the application of agroforestry and soil conservation techniques in tropical highlands. Working Paper No. 41. International Council for Research in Agroforestry (ICRAF), Nairobi, Kenia.

Voroney, R.P., J.A. van Veen \& E.A. Paul. 1981. Organic carbon dynamics in grassland soils. II. Model validation and simulation of the long-term effects of cultivation and rainfall erosion. Can. J. Soil. Sci. 6: 211-214.
Warren, G. 2001. Environmental weeds of the wet tropics bioregion: risk assessment and priority ranking. Report for the Wet Tropics Management Authority, Cairns, Australia.

Wynn, T. \& S. Mostaghimi. 2006. The effects of vegetation and soil type on streambank erosion, southwestern Virginia, USA. J. Am. Water Resour. As. 42: 69-82.

Wynn, T. 2006. Streambank retreat: a primer. AWRA Hydrology \& Watershed Management Technical Committee. Watershed Update 4(1): 1-14 (también disponible en línea: http://www.nueces-ra.org/CP/ LS/pdfs/svp.pdf).

Wynn, T., S. Mostaghimi, J. Burger, A. Harpold, M. Henderson \& L.-A. Henry. 2004. Variation in root density along streambanks. J. Environ. Qual. 33: 2030-2039.

Zaimes, G.N., R.C. Schultz, T.M. Isenhart, S.K. Mickelson, J.L. Kovar, J.R. Russell \& W.P. Powers. 2005. Stream bank erosion under different riparian land-use practices in northeast Iowa. In Proceedings of the 9th North American Agroforestry Conference, June 12-15, 2005 (2005 CD-ROM), Rochester, Minesota, EEUU.

Zar, J.A. 1984. Biostatistical Analysis. Prentice-Hall, Englewood Cliffs, Nueva Jersey, EEUU.

\section{REFERENCIAS DE INTERNET}

Anónimo. 1999. Definición de "humedales" y sistema de clasificación de tipos de humedales de la Convención de Ramsar. (Consultado el 16 de diciembre del 2008, http://www.ramsar.org/ris/key_ris_types_s.htm). 
